\title{
A introdução do complexo econômico industrial da saúde na agenda de desenvolvimento: uma análise a partir do modelo de fluxos múltiplos de Kingdon
}

\author{
Antoine Metten \\ Fundação Oswaldo Cruz (Fiocruz) / Grupo de Pesquisa de Inovação em Saúde (GIS) \\ Rio de Janeiro / RJ - Brasil \\ Laís Silveira Costa \\ Fundação Oswaldo Cruz (Fiocruz) / Grupo de Pesquisa de Inovação em Saúde (GIS) \\ Rio de Janeiro / RJ - Brasil \\ Carlos Augusto Grabois Gadelha \\ Ministério do Desenvolvimento, Indústria e Comércio Exterior (MDIC) \\ Rio de Janeiro / RJ - Brasil \\ Jose Maldonado \\ Fundação Oswaldo Cruz (Fiocruz) / Escola Nacional de Saúde Pública Sergio Arouca \\ Rio de Janeiro / RJ - Brasil
}

\begin{abstract}
O complexo da saúde refere-se a toda a base produtiva envolvida na prestação de serviços, promoção e vigilância em saúde, envolve tanto o segmento secundário da economia, a exemplo das indústrias de base química, biotecnológica, mecânica e de materiais, quanto o terciário, referente aos serviços prestados nos estabelecimentos de saúde. O referido complexo mobiliza uma série de tecnologias relevantes para a inserção competitiva na economia global, responde pela geração de emprego, renda e parte significativa do PIB nacional, motivo pelo qual a área da saúde tem tido sua importância crescentemente institucionalizada na agenda de desenvolvimento nacional. Este artigo propõe analisar a introdução desse complexo nas prioridades do Plano Brasil Maior, dado que a saúde responde a diferentes elementos relacionados tanto a uma conjuntura política quanto à escolha dos tomadores de decisão entre variadas opções de políticas públicas, como também aos problemas socioeconômicos observados na referida área. Esses distintos elementos serão estudados à luz do referencial teórico de fluxos múltiplos, utilizando como método de análise a confrontação dos fluxos de problemas, soluções e político. Os principais resultados do estudo apontam que a decisão de incorporar o complexo econômico industrial da saúde nas prioridades do Plano Brasil Maior é fruto de uma abordagem renovada da saúde,
\end{abstract}

DOI: http://dx.doi.org/10.1590/0034-7612123873

Artigo recebido em 17 set. 2013 e aceito em 27 jan. 2014.

(cc) BY-NC

Rev. Adm. Pública - Rio de Janeiro 49(4):915-936, jul./ago. 2015 
tanto no nível cognitivo quanto no institucional, na qual a mesma é entendida simultaneamente como um direito do cidadão, um bem econômico e social assim como um espaço de acumulação de capital.

Palavras-chave: complexo econômico industrial da saúde; Plano Brasil Maior; modelo de fluxos múltiplos; instituições; política.

La introducción del complejo de la salud en la agenda de desarrollo: un análisis desde el modelo de flujos múltiples de Kingdon

El complejo de la salud se refiere a la totalidad de la base de producción involucrada en la prestación de servicios, promoción y vigilancia de la salud y moviliza tanto el segmento secundario de la economía, como las industrias química, biotecnología, mecánica y de materiales, como el terciario, referente a los servicios prestados en materia de salud. Tal complejo moviliza una serie de tecnologías relevantes para la inserción competitiva en la economía global, responde mediante la generación de empleo, ingresos, y de una parte significativa del PIB nacional, explicando porque la importancia de la salud se ha institucionalizado cada vez más en la agenda nacional de desarrollo. Este artículo tiene como objetivo analizar la introducción de este complejo en las prioridades del Plan Mayor Brasil, dado que responde a diferentes factores relativos tanto a la situación política en cuanto a la elección de los tomadores de decisiones entre muchas opciones de políticas públicas, sino también a problemas socioeconómicos observados en la salud. Estos diferentes elementos se estudiarán a la luz del marco teórico de múltiples flujos, como un método de análisis mediante la comparación de los flujos de problemas, soluciones y político. Los principales resultados del estudio indican que la decisión de incorporar el complejo económico industrial de la salud en las prioridades de el Plan Brasil Mayor es el resultado de una percepción renovada de la salud, tanto en el nivel cognitivo como institucional, en que se entiende tanto como un derecho de los ciudadanos, un bien económico y social así como un espacio para la acumulación de capital.

Palabras clave: complejo económico industrial de la salud; Plan Brasil Mayor; modelo de múltiples flujos; instituciones; política.

The introduction of the health economic industrial complex in the development agenda: an analysis from Kingdon's multiple streams model

The health care complex refers to all productive base involved in service provision, promotion and health surveillance. It involves both the secondary sector of economy, as the chemical-based industries, biotechnology, mechanics and materials, and the tertiary, which includes services rendered in health establishments. Such complex mobilizes a series of technologies relevant to the competitive insertion in the global economy, represents a significant part of the national GDP and it is a source of jobs and income, which is why health care economic importance has been increasingly institutionalized in the national development agenda. This article aims to analyze the introduction of this complex in Brasil Maior Plan priorities, given that health responds to different factors pertaining to both the political situation and the choice made by decision makers among many options of public policies, but also to socioeconomic problems observed in health. Those elements will be studied through the use of the theoretical framework of multiple streams model, using as a method of analysis the confrontation of streams of problems, solutions and macro political context. The main results of the study indicate that the decision to incorporate the health economic industrial complex to Brasil

Rev. Adm. Pública - Rio de Janeiro 49(4):915-936, jul./ago. 2015 
Maior Plan priorities is the result of a renewed approach of health care, both at the cognitive and institutional level, in which it is understood as a citizen's right, an economic and social good as well as a space for capital accumulation.

Keywords: health economic industrial complex; Brasil Maior Plan; sample multiple streams; institutions; politics.

\section{Introdução}

A sustentabilidade do sistema de saúde remete ao padrão de desenvolvimento nacional, uma vez que a mesma depende de características da população (demografia, nível e distribuição da renda, emprego, grau e qualidade da urbanização, entre outros determinantes sociais) e que sua base produtiva apresenta grande potencial econômico em termos de geração de renda, empregos qualificados e inovações (Gadelha, Maldonado e Costa, 2012). A saúde possui, dessa forma, duas dimensões: uma de política de proteção social e outra de fonte de riqueza para o país. Logo, as políticas de saúde têm que considerar essas duas dimensões, ou seja, é necessário que a ação pública procure integrar a política social à política industrial, e vice-versa.

É a partir desse entendimento que o complexo econômico-industrial da saúde (ou complexo da saúde) foi designado como um dos setores prioritários para o desenvolvimento nacional, no âmbito do Plano Brasil Maior. Lançado no dia 8 de fevereiro de 2011, com o slogan "Inovar para competir. Competir para crescer.", o referido Plano constitui um dos pilares da política industrial, tecnológica e de comércio exterior do governo federal na atualidade (Brasil, 2011). Designar a base produtiva da saúde como uma das prioridades dessa política traduz, portanto, o reconhecimento institucional do potencial da mesma para a geração de renda, empregos e inovações, além de seu já reconhecido papel no fortalecimento do estado de bem-estar.

Concretamente, a incorporação do complexo da saúde nas prioridades do Plano Brasil Maior se desdobra em uma série de medidas para fortalecer sua capacidade produtiva e de geração e difusão do progresso técnico. Essas medidas dizem respeito: 1) ao fortalecimento do parque produtivo de fármacos, medicamentos, equipamentos e materiais de uso em saúde no País; 2) à utilização do poder de compra governamental para aquisição de produtos e serviços estratégicos ${ }^{1}$ para o Sistema Único de Saúde (SUS); 3) ao fortalecimento da produção pública mediante a qualificação da gestão e ampliação de investimentos ${ }^{2}$ para atender às demandas

\footnotetext{
${ }^{1}$ As licitações para compra de determinados produtos podem se beneficiar de uma margem de preferência de até $25 \%$ em função do potencial de geração de emprego e renda e de inovação tecnológica dos mesmos para o país. Para mais detalhes ver Decreto no 7.713, de 3 de abril de 2012, que emenda a Lei no 12.349/2012.

${ }^{2}$ A política envolve a realização de parcerias para o desenvolvimento produtivo, via transferência de tecnologia entre laboratórios internacionais e públicos, de modo a qualificar e aumentar a produção pública de fármacos e medicamentos.
}

Rev. Adm. Pública - Rio de Janeiro 49(4):915-936, jul./ago. 2015 
do SUS e 4) à prospecção e monitoramento tecnológico para orientar a política de inovação visando ao acesso universal aos produtos para saúde.

Dessa forma, a decisão de incluir a base produtiva da saúde nas prioridades do Plano reflete a institucionalização de uma visão sistêmica da mesma, entendendo-a também como fator de dinamismo econômico e tecnológico e produtor dos insumos necessários à universalização do acesso aos serviços de saúde. Ao mesmo tempo, essa decisão é o resultado de um processo interno às instituições envolvidas, tributário da influência de grupos organizados dentro e fora dessas instituições —, da avaliação de políticas públicas anteriores, da definição do problema e da formulação de soluções, assim como do contexto macropolítico no qual ela se insere (Lascoumes e Le Galès, 2012).

Em decorrência disso, objetiva-se, neste estudo, compreender como esse tema foi introduzido no conjunto de preocupações dos formuladores de políticas, transformando-se em uma política pública. Para tanto, analisar-se-ão os condicionantes político-institucionais relacionados à confrontação entre os problemas identificados na área da saúde, o leque de soluções disponíveis para sua superação e o contexto macropolítico que tornaram possível a incorporação do complexo econômico-industrial da saúde nas prioridades da agenda de desenvolvimento nacional pautada pelo Plano Brasil Maior.

A abordagem teórica utilizada para analisar o processo de tomada de decisão pública (modelo de fluxos múltiplos) será objeto da segunda parte, e a terceira parte trata especificamente do processo de inclusão do complexo econômico industrial da saúde (Ceis) nas prioridades do Plano Brasil Maior, sendo seguida de considerações finais.

\section{0 processo de tomada de decisão pública}

O estudo do processo de tomada de decisão pública abarca o conjunto dos elementos que conferem a fatos sociais o status de problema público. Dessa forma, eles deixam de depender da fatalidade (natural ou social) ou da esfera privada para se tornarem objetos de debates e controvérsias midiáticas e políticas, de maneira a definir coletivamente a melhor forma de resolvê-los (Augusto, 1989).

Entretanto, o âmbito do que se qualifica como público, ligado ao interesse geral, não é delimitado de maneira isenta; isso porque o Estado é um lugar de domínio e de conflitos, contraditório em sua natureza, e não um espaço de neutralidade, situado além e acima das diferenças constitutivas do social. Nesse processo, o Estado tende a se constituir como sujeito personificado e personalizável na dinâmica social, produzindo bens e serviços de interesse coletivo ou outorgando direitos e benesses aos diferentes segmentos sociais (Augusto, 1989:1).

Cabe destacar que o campo teórico da policy analysis possui três conceitos fundamentais, conforme lembram Flexor e Leite (2006). O primeiro refere-se à Polity, que diz respeito à dimensão institucional do sistema político, delineada pelo sistema jurídico e o sistema político-administrativo; o segundo, à Politics, que diz respeito ao quadro da dimensão processual, tendo como foco o processo político em si, frequentemente de caráter conflituoso; e, por fim, 
o terceiro, à Policy, que remete à dimensão material, ou seja, os conteúdos concretos do sistema, que correspondem à configuração dos programas políticos, aos problemas técnicos e ao conteúdo material das decisões políticas.

Estudos de análise de políticas públicas foram desenvolvidos de forma sistemática a partir dos anos 1950, com o objetivo de entender a natureza dessas distintas influências sobre o processo de tomada de decisão na formulação e implementação da ação pública. Em particular, a leitura neoinstitucionalista em ciência política (Taylor e Hall, 2003, apud Flexor e Leite, 2006) e a sociologia das organizações revelam que a repartição dos recursos (financeiros, institucionais ou simbólicos) efetua-se de maneira assimétrica entre os grupos sociais representados dentro das instituições públicas, condicionando o acesso desproporcional dos mesmos ao processo de decisão política. Nesse processo, cabe destacar a influência das redes de política pública ${ }^{3}$ e das coalizões de defesa, ${ }^{4}$ uma vez que as mesmas têm como objetivo justamente influenciar os resultados finais da ação pública. Nesse sentido, saliente-se o conceito de referencial de política pública, definido como o "jogo das representações sociais imediatamente associadas à negociação de políticas públicas” (Jobert, 1995:153), dividido entre os membros de uma determinada rede, ou, no vocabulário do autor, uma comunidade de política pública. Esse referencial, ou "uma imagem da realidade sobre a qual queremos intervir" (Jobert, 1995:164), tem grande influência sobre a atuação de uma dada comunidade de política pública, uma vez que norteia o entendimento dos condicionantes do problema a tratar, os quais definem, por sua vez, um leque de soluções adequadas ao seu tratamento. Dessa forma, o referencial de política pública orienta o comportamento dos tomadores de decisão na hora da escolha entre as distintas soluções existentes para o tratamento de um determinado problema. A escolha entre os papéis dentro da "lata de lixo"5 (Cohen, March e Olsen, 1972) não é neutra.

Um dos focos da análise de políticas e programas governamentais encontra-se no estudo de uma ou mais fases que compõem, de acordo com a percepção heurística da análise de política pública, o ciclo de execução de uma política pública (policy cicle). Frey (2000), retomando a conceptualização proposta originalmente por Jones (1970), resumiu essas fases como: a) determinação da agenda, onde a dinâmica da definição do problema é questão essencial para a compreensão da política pública; b) formulação e legitimação da política

\footnotetext{
3 "Uma rede de política pública é o resultado de uma cooperação mais ou menos estável e hierárquica entre organizações que negociam, trocam recursos e podem compartilhar normas e interesses num ambiente complexo" (Flexor e Leite, 2006:14).

4 "Uma coalizão de defesa pode ser definida como um conjunto de atores (de organizações públicas, de todos os níveis de governo, e da sociedade civil) que compartilham convicções e objetivos políticos e que almejam administrar as regras das diversas instituições para atingir seus objetivos no decorrer do tempo" (Sabatier e Weible, 2007, apud Gomide, 2008:20).

${ }^{5}$ Cohen, March e Olsen (1972) usam a metáfora da "lata de lixo" para mostrar que a racionalidade dos formuladores de política é limitada: os distintos problemas e soluções assemelham-se a papéis jogados numa lata lixo de forma aleatória e, em um dado momento, os tomadores de decisão escolhem uma solução para um determinado problema. A racionalidade dos atores é limitada pelo fato de que a ação precede a definição das preferências e que a ação é subordinada à energia que os mesmos estão dispostos a investir na aplicação da ação em questão.
} 
(seleção de proposta, construção de apoio político, formalização em lei); c) implementação de políticas (operacionalização da política em planos, programas e projetos no âmbito da burocracia pública e sua execução); d) e avaliação de políticas (relato dos resultados alcançados com a implementação das propostas e programas de governo, avaliação dos impactos dos programas e sugestão de mudanças). É reconhecido o papel das redes de política pública em cada fase do processo, uma vez que as mesmas procuram influir sobre a introdução (ou não) de um problema na agenda política, sobre a formulação e/ou implementação da própria política, assim como sobre sua avaliação, de maneira a defender a continuidade (ou a extinção) de uma determinada política pública.

Apesar de pioneira, essa abordagem sequencial da análise de políticas públicas (policy analysis) vem sendo criticada ${ }^{6}$ com base em diversos estudos empíricos acerca da implementação de políticas públicas. Majoritariamente, as críticas referem-se ao fato de a abordagem ser bastante esquemática e de sua incapacidade de retratar e analisar situações que reflitam simultaneamente duas ou mais fases do ciclo, conforme observado em numerosos estudos de casos de políticas públicas. A despeito disso, ela ainda constitui uma referência útil para situar o estágio de desenvolvimento de uma política pública sobre o qual uma determinada análise pretende se concentrar. No presente estudo, pretende-se analisar o processo de tomada de decisão na fase de determinação da agenda governamental (fase "a"), reconhecendo que não existe separação estanque entre as distintas fases de execução da ação pública, ou seja, que a fase de determinação da agenda sofre influência daquela de implantação e de avaliação de programas anteriores tal como integra elementos respondendo à sua futura formulação. No plano da dinâmica política, a formação da agenda resulta de um conjunto de fatores que envolvem tanto o momento específico de mudança na gestão quanto as características do projeto político do governo, como também o contexto macropolítico favorável (ou não) a um determinado projeto político (Capella, 2008:5).

Com base nesse entendimento, apresenta-se em seguida o referencial analítico utilizado nessa investigação, composto pelo modelo de fluxos múltiplos, elaborado por John W. Kingdon em 2003. O recurso a esse arcabouço teórico justifica-se porque tal ferramenta analítica permite identificar os elementos de natureza socioeconômica, política e institucional que configuram os determinantes da tomada de decisão que levam à introdução da base produtiva na política de desenvolvimento nacional, renunciando voluntariamente à análise detalhada de sua formulação, implementação e avaliação. ${ }^{7}$ Objetiva-se avançar no esclarecimento da seguinte questão: "por que alguns problemas se tornam importantes para um governo? Como uma ideia se insere no conjunto de preocupações dos formuladores de políticas, transformando-se em uma política pública?" (Capella, 2012:87-88).

\footnotetext{
${ }^{6}$ Para uma leitura crítica do modelo sequencial de Jones incluindo, entre outras críticas, a hipótese de não decisão, o reconhecimento de fronteiras flexíveis entre as sequências, a crítica à abordagem de problem solving, ou a não incorporação do papel da dimensão simbólica na formulação de política pública, ver, por exemplo, Jobert e Muller (1987). ${ }^{7} \mathrm{O}$ arcabouço teórico dos fluxos múltiplos é utilizado neste estudo em função de o mesmo propiciar a identificação e análise dos elementos político-institucionais centrais ao processo de tomada de decisão que se pretende estudar (problemas, soluções e contexto macropolítico).
} 


\section{0 modelo de fluxos múltiplos}

Dentre as matrizes de análise de políticas públicas existentes, o modelo de fluxos múltiplos (multiple streams framework, Kingdon, 2003), cuja primeira formulação data dos anos 1970 visando analisar as políticas de saúde e transporte nos EUA, vem ganhando expressão desde os anos 2000 em distintos estudos de casos, notadamente no Brasil. Em termos gerais, o modelo é utilizado para explicar as decisões e definições de agendas de políticas públicas por governos nacionais. Nesse sentido, o modelo busca entender por que alguns problemas se tornam importantes para um governo, a ponto de os governantes aceitarem uma ideia dentre um conjunto de preocupações existentes e transformá-la em uma política pública. Estudos como os de Nagem e Silva (2011), Capella (2006, 2008, 2012), Abreu, Neiva e Lima (2012) e Gomide (2008) contribuíram para aprofundar o modelo de Kingdon ao utilizar essa matriz teórica na análise de algumas políticas públicas brasileiras.

Para Kingdon (2003), a agenda governamental é definida a partir de um conjunto de temas ou problemas em pauta sob um determinado momento que demanda a atenção da equipe de governo e seus assessores. No entanto, dada a grande variedade de questões em pauta, somente algumas delas serão de fato consideradas e inseridas na agenda de ação governamental (agenda-setting), ou seja, serão objeto de decisão coletiva e determinarão procedimentos e especificação de alternativas.

A definição de temas prioritários em uma agenda governamental é afetada por processos pelos quais determinados itens da agenda conseguem o destaque necessário, levando à sua institucionalização e posterior implementação. De acordo com o modelo de fluxos múltiplos (Kingdon, 2003), esses processos envolvem três fluxos decisórios (streams), a saber: 1) problemas (problems), que podem ser identificados por meio de indicadores, crises ou feedback de outras ações em curso ou concluídas; 2) soluções ou alternativas (policies), que são debatidas em comunidades específicas de especialistas e são definidas a partir de sua viabilidade técnica e financeira; e 3) político (politics), que envolve o ambiente macropolítico, ou "humor nacional" (national mood), caracterizado por uma situação em que diversas pessoas compartilham as mesmas questões durante um determinado período, as forças políticas organizadas que determinam o apoio ou oposição a uma questão em pauta e as mudanças na alta administração do governo (Capella, 2012).

De acordo com a autora, há uma relativa independência entre os três fluxos, que se desenvolvem segundo suas próprias dinâmicas. A convergência dos três fluxos em um dado momento abre uma "janela de oportunidade política" no processo de definição de agenda e possibilita a introdução de um novo item na política pública.

Outro componente importante no modelo de Kingdon é a atuação dos chamados "empreendedores políticos", situados no governo (no Poder Executivo, ocupando altos postos ou em funções burocráticas; no Congresso, sejam parlamentares ou assessores), ou fora dele (em grupos de interesse, na comunidade acadêmica, na mídia) que estão sempre prontos para atuar no momento em que uma janela política se abre. Em geral, são especialistas que gozam de boa reputação, habilidade de negociação ou detêm certa popularidade política. Além disso,

Rev. Adm. Pública - Rio de Janeiro 49(4):915-936, jul./ago. 2015 
estão dispostos a investir recursos como tempo, energia e dinheiro para promover uma ideia e influenciar o processo de formulação de políticas, unindo soluções a problemas. Os principais incentivos que os movem vão desde interesses ideológicos e promoção de valores que lhes são caros até a busca por benefícios materiais ou uma maior importância na esfera de poder. Assim, os empreendedores desempenham um papel fundamental, vasculhando a "lata de lixo" (Cohen, March e Olsen, 1972) e unindo: soluções a problemas; propostas a momentos políticos; eventos políticos a problemas. Sem um empreendedor, essa ligação entre os fluxos pode não acontecer, ou seja, ideias não serão defendidas; problemas não encontrarão soluções; ou, ainda, observarse-á a perda de momentos políticos favoráveis à mudança por falta de propostas.

Por fim, a influência presidencial recebe especial destaque no modelo de fluxos múltiplos. Por manejar recursos institucionais (principalmente o poder de veto e o poder de nomear pessoas para cargos-chave no processo decisório), recursos organizacionais e recursos de comando da atenção pública, o presidente é considerado por Kingdon (2003) o ator mais forte na definição da agenda. A figura 1 esquematiza os conceitos-chave do modelo de fluxos múltiplos.

Figura 1

O modelo de fluxos múltiplos (Kingdon, 2003)

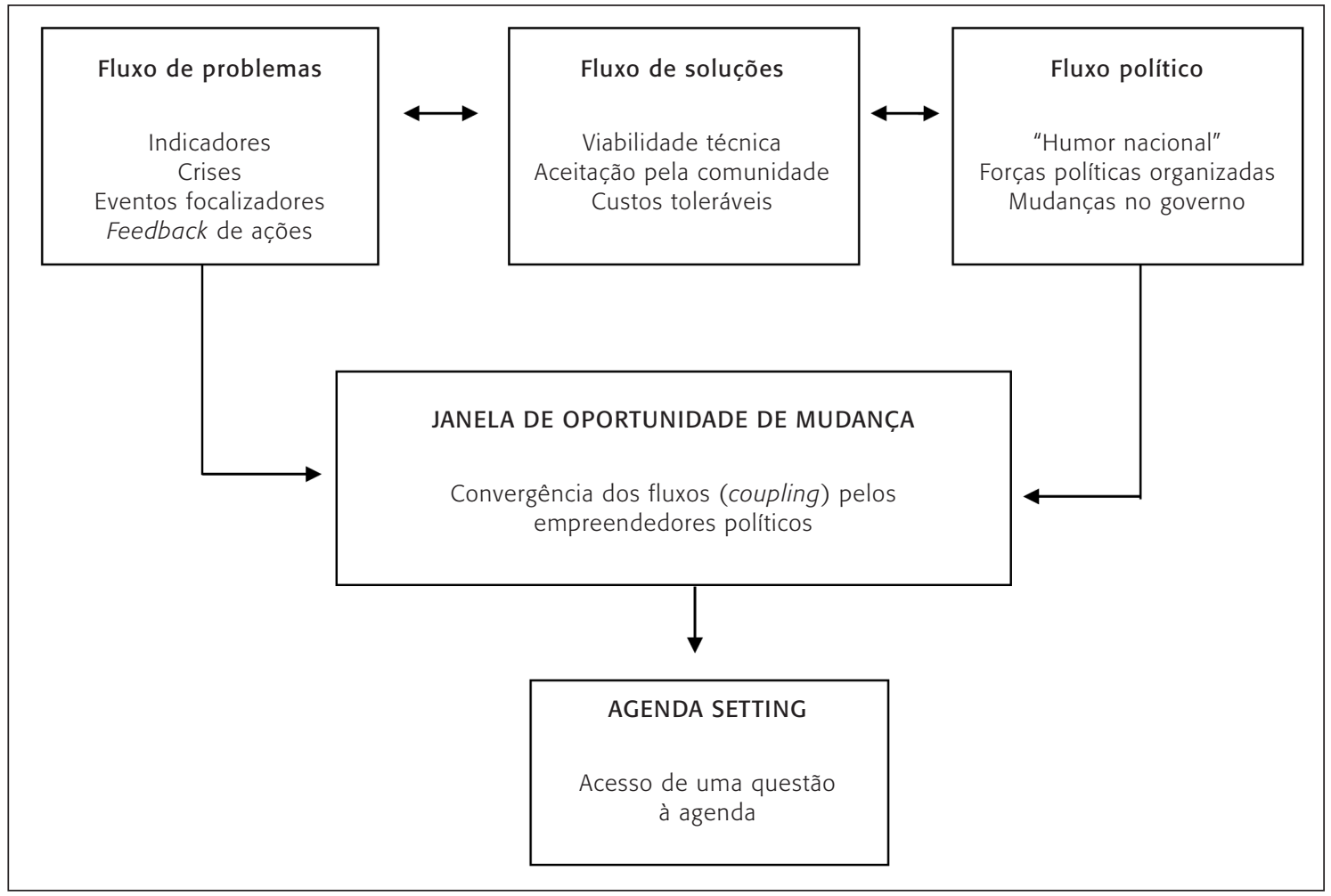

Fonte: Capela (2012:98). 
Cabe destacar que a existência de um contexto político favorável não garante, por si só, a colocação de um problema na agenda governamental. Um contexto político pode estimular, ao mesmo tempo, diversas ideias e esse elemento, tomado isoladamente, não explica o porquê da ascensão de uma questão específica. É preciso, adicionalmente à existência desse contexto favorável, que problemas sejam definidos, representados estrategicamente e comunicados de forma a sensibilizar diversos atores para a urgência e oportunidade da ação governamental. Assim, de acordo com Capela (2012:105):

O modelo focaliza a dinâmica das ideias: o desenvolvimento de políticas é visto como uma disputa sobre definições de problemas e geração de alternativas. Rompendo com esquemas interpretativos deterministas, o modelo assume uma lógica contingencial. A mudança na agenda depende da combinação entre problemas, soluções e condições políticas. A existência de um problema não determina a adoção de uma solução específica e não cria por si só um ambiente político favorável para a mudança.

Da mesma forma, é preciso também que soluções sejam difundidas, discutidas, apresentadas em diversos fóruns, mobilizando grupos distintos. Um contexto favorável sem a difusão de uma representação de problemas e de soluções desejáveis não promove mudanças na agenda. Para retomar o vocabulário de Jobert (1995), é necessário comunicar amplamente o referencial da ação pública para lhe conferir legitimidade. Com baixa visibilidade, o problema tende a ser colocado em segundo plano no processo decisório e, consequentemente, não gera oportunidades para que uma questão integre a agenda governamental (Capella 2008:12).

A partir desse entendimento do processo de tomada de decisão pública é possível identificar de forma sistematizada os elementos que condicionaram a colocação do complexo econômico-industrial da saúde nas prioridades do Plano Brasil Maior.

\section{A introdução do complexo econômico industrial da saúde nas prioridades do Plano Brasil Maior}

A introdução do complexo da saúde nas prioridades da agenda do Plano Brasil Maior não representa em si uma inovação na ação pública nacional. O caráter estratégico desse complexo já havia sido estabelecido em distintos programas a partir dos anos 2000. A Política Industrial e Tecnológica e de Comércio Exterior, lançada em março de 2004, foi pioneira nesse aspecto, ao reconhecer o potencial da cadeia produtiva farmacêutica para alavancar a incorporação de tecnologias portadoras de futuro (nanotecnologia, biotecnologia e química fina, entre outras). Em 2007, no âmbito do Programa Mais Saúde foi reconhecida a necessidade de fortalecimento da dinâmica produtiva do complexo da saúde para reduzir a dependência aos mercados externos de medicamentos e material médico hospitalar, e, portanto, a vulnerabilidade da política de saúde brasileira. Por sua vez, em 2008, a Política de Desenvolvimento Produtivo, a partir do estabelecimento de metas quantitativas, priorizou o complexo da saúde visando

Rev. Adm. Pública - Rio de Janeiro 49(4):915-936, jul./ago. 2015 
estimular a geração de conhecimento científico-tecnológico para a redução da vulnerabilidade do sistema nacional de saúde.

A decisão de incluir o complexo econômico-industrial da saúde nas prioridades do Plano Brasil Maior não refletiu, portanto, uma ruptura na trajetória do estabelecimento da ação pública voltada à produção industrial na área da saúde para o desenvolvimento nacional, mas sim refletiu continuidade do reconhecimento do caráter sistêmico desse complexo, capaz de articular desenvolvimento econômico, dinamismo tecnológico e fortalecimento do Estado de bem-estar social. Entretanto, seu ingresso nas prioridades do principal programa de desenvolvimento do país constitui um marco importante no reconhecimento institucional do caráter estratégico do complexo da saúde para o desenvolvimento nacional. De acordo com a teoria dos fluxos múltiplos supracitada, esse processo se deu graças à junção de elementos ligados à percepção do papel da saúde no projeto de desenvolvimento nacional oriundos de três fluxos: problemas, soluções e político, os quais serão expostos a seguir.

\subsection{0 fluxo de problemas}

Assim, recorrendo à teoria dos fluxos, a definição do problema resulta da análise de uma série de indicadores, assim como de feedbacks de ações empreendidas para fortalecer a base produtiva da saúde.

$\mathrm{Na}$ análise do fluxo de problemas da saúde, ressalta-se a fragilidade de sua base produtiva nacional, no que diz respeito à sua capacidade de inserção nos mercados internacionais de fármacos, medicamentos e de equipamentos médico-hospitalares mais complexos, assim como suas limitações para atender as necessidades sanitárias da população.

Com efeito, a expansão do acesso aos cuidados de saúde observada nos últimos anos levou a um crescimento substantivo do déficit da balança comercial do complexo da saúde. De acordo com o gráfico 1, o mesmo mais que triplicou na última década, passando de um patamar de US\$ 3 bilhões em 2003 para mais de US\$ 11 bilhões em 2013.

Essa situação evidencia a debilidade da base produtiva nacional da saúde e sua dependência em relação aos mercados internacionais, justificando assim a necessidade de elaboração de políticas públicas voltadas para o apoio à produção nacional dos insumos estratégicos necessários para o funcionamento do sistema de saúde (Gadelha, Costa e Viana, 2011).

A busca de autonomia da base produtiva nacional é particularmente relevante no caso da saúde, dado o caráter assimétrico e oligopolístico dos mercados internacionais das indústrias farmacêutica e de equipamentos e materiais hospitalares e odontológicos (Maldonado, 2012; Vargas, 2012). Com efeito, de acordo com Gadelha e colaboradores (2012), verificase que as 10 maiores empresas farmacêuticas do mundo - todas elas oriundas dos Estados Unidos ou da União Europeia - totalizavam 34\% do total das vendas mundiais em 1999 e cerca de $45 \%$ em 2010. Da mesma forma, no que diz respeito ao mercado internacional de equipamentos e materiais hospitalares e odontológicos, constata-se que, das 10 empresas que mais investiram em pesquisa e desenvolvimento em 2010, nove eram estadunidenses e uma 
japonesa. Em 2013, os Estados Unidos e a Europa Ocidental totalizavam mais de 70\% do mercado mundial (The World Medical Markets Fact Book, 2013).

\section{Gráfico 1}

Evolução da balança comercial do complexo econômico-industrial da saúde 1996 - 2013 (valor em US\$ bilhões, atualizado pelo IPC/EUA)

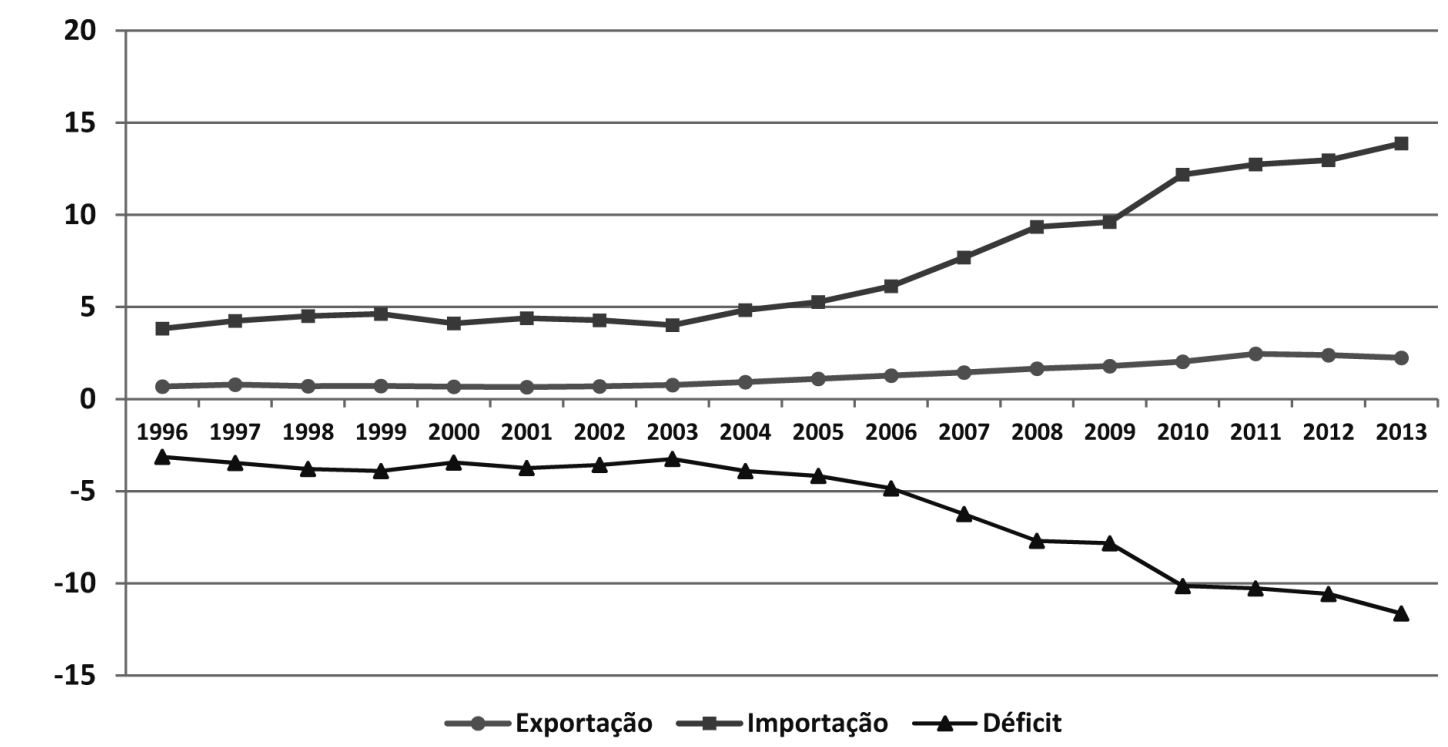

Fonte: Elaborado por GIS/Ensp/Fiocruz, 2014, a partir de dados da Rede Alice/MDIC. Disponível em: <http://aliceweb.mdic.gov.br>. Acesso em: 10 fev. 2014.

Esses fatores revelam a fragilidade da base produtiva em saúde, com impacto, inclusive, na autonomia nacional sobre a política de saúde, uma vez que limites são impostos à mesma em função da taxa cambial, das relações internacionais e da influência limitada na escolha das prioridades da agenda de pesquisa e desenvolvimento em tecnologias de saúde.

Assim, em função de a estrutura produtiva do Complexo ser caracterizada como um oligopólio mundial intensivo em ciência e tecnologia, articulando concentração industrial, lucros excepcionais, barreiras tecnológicas à entrada e combinação de crescimento no consumo com aumento de preços, entre outros, observa-se uma combinação de fatores que dificulta a entrada de novos concorrentes, pelo menos no que se refere aos produtos de maior valor agregado (Gadelha et al., 2012). Considerando a baixa capacidade produtiva nacional, as indústrias do país, quando competitivas, limitam sua atuação a determinados nichos de mercado de menor intensidade tecnológica, onde opera uma concorrência em termos de escala de produção. Nessas condições, o aumento da oferta dos serviços, especialmente a partir da promulgação da Carta Magna, quando se estabeleceu a universalidade da oferta da saúde, intensificada com a melhoria das condições socioeconômicas da população brasileira e o quadro global de en- 
velhecimento da população, foi acompanhado de um aumento substantivo da importação de insumos, fato que reflete fortemente no já mencionado déficit da balança comercial do Ceis, ameaçando a soberania nacional na política de saúde e a viabilidade do SUS como um todo, um dos maiores sistemas de saúde universal do mundo e, no Brasil, principal ícone da política social nacional e da própria estrutura do estado de bem-estar.

Do ponto de vista econômico, cabe mencionar a necessidade de superar a defasagem do parque industrial brasileiro ${ }^{8}$ assim como proporcionar ganhos substanciais quanto à articulação do desenvolvimento científico e tecnológico com a indústria nacional (Gadelha e Costa, 2012). Da mesma forma é necessário adequar o marco regulatório à necessidade de incorporação no SUS dos medicamentos requeridos, em função das necessidades da população. Cabe destacar que o prazo médio de aprovação de um remédio por parte da Agência Nacional de Vigilância Sanitária (Anvisa), afetada por uma estrutura de recursos humanos subdimensionada, chega no final de 2012 a 640 dias, contra um máximo de 180 dias para produtos considerados estratégicos nos Estados Unidos (O Estado de S. Paulo, 26 nov. 2012).

Nessas condições, evidencia-se a necessidade de formular e implementar políticas públicas voltadas para o fortalecimento das indústrias do Ceis, envolvendo incentivos à inovação em saúde, estabelecimento de um marco regulatório adequado às necessidades da base produtiva nacional e uso do poder de compra do Estado. De acordo com Cimoli e colaboradores (2007:60):

(...) em todos os países dotados de indústrias farmacêuticas eficientes e com fins lucrativos encontramos programas governamentais de apoio à pesquisa biomédica, geralmente em universidades e laboratórios públicos. Ao mesmo tempo, os participantes de tais programas pelo lado das universidades estão também associados à capacitação científica de pessoal que, após o término de sua formação, irá trabalhar nas empresas farmacêuticas. Além disso, praticamente em todos os países, fundos e programas públicos desempenham um papel importante nas compras governamentais de produtos farmacêuticos. E, finalmente, em quase todos os países há diversas formas de regulamentação dos mercados de produtos farmacêuticos que vão muito além das garantias de direitos de propriedade e de honestidade das transações previstas pelos manuais.

Assim, os problemas que levaram à inserção do complexo econômico-industrial da saúde nas prioridades do Plano Brasil Maior basearam-se essencialmente no esforço de fortalecimento do estado de bem-estar social, assim como na necessidade de promover a inserção competitiva internacional. Para enfrentar esses desafios, os formuladores de políticas públicas tiveram que procurar soluções e alternativas inovadoras.

\footnotetext{
${ }^{8}$ A defasagem do parque industrial nacional remete ao debate contemporâneo sobre a desindustrialização brasileira e a necessidade de tornar competitivo o parque industrial brasileiro, fruto do reconhecimento de que a indústria continua sendo um elo estratégico para o uso, geração e difusão de inovação que impacta todo o sistema produtivo. Para mais detalhes, ver Carvalho e Kupfer (2011).
} 


\subsection{0 fluxo de soluções}

O fluxo de soluções e alternativas caracterizou-se pela emergência de uma abordagem renovada da saúde, a qual contempla um entendimento mais sistêmico da mesma, em que se reconhece que o padrão e o estágio de desenvolvimento de uma dada sociedade impactam as condições de vida da população (determinantes sociais da saúde). De forma análoga, a saúde passa a ser reconhecida como importante indutor de desenvolvimento, não somente por sua importância social, como também por seu papel na geração de emprego, renda e fonte de progresso técnico para o país, uma vez que representa aproximadamente $9 \%$ do PIB, $10 \%$ dos empregos qualificados e 30\% do esforço em Pesquisa e Desenvolvimento (Gadelha e Costa, 2012). Nessa perspectiva, o conceito de desenvolvimento é entendido como um "processo de mudança social pelo qual o crescente número de necessidades humanas (...) são satisfeitas através de uma diferenciação no sistema produtivo, gerado pela introdução de inovações tecnológicas" (Furtado, 1964, apud Guillén, 2007:143). É reconhecido, dessa forma, que a saúde articula um complexo de atividades produtivas que relacionam segmentos cruciais da sociedade do aprendizado (baseadas no conhecimento e na inovação), identificando-a com fatores de competitividade global (Guimarães, 2006; Albuquerque e Cassiolato, 2000; Lastres e Cassiolato, 2007). Com base nesse entendimento sistêmico da saúde, alguns atores se envolveram na defesa de uma política de produção nacional de insumos de saúde. Entre eles, é possível destacar - sem pretensão à exaustividade - a atuação, no campo institucional, do Grupo Executivo do Complexo Industrial da Saúde ${ }^{9}$ constituído por representantes de setores diversos do governo, coordenado pelo Ministério da Saúde, o qual também administra o Fórum Permanente de Articulação com a Sociedade Civil. Além desses grupos especializados, pode-se citar a atuação do Departamento de Prioridades, ligado à Área de Planejamento do Banco Nacional de Desenvolvimento Econômico e Social (BNDES), responsável pelo programa Profarma, desenvolvido para financiar investimentos destinados a alavancar o aumento de competitividade do Ceis e à redução da vulnerabilidade externa do sistema de saúde nacional. Por último, vale mencionar a atuação do Ministério da Ciência e Tecnologia e Inovação, que vem reconhecendo o caráter crítico de inversão em ciência e tecnologia na base produtiva da saúde, e da Diretoria de Inovação da Financiadora de Estudos e Projetos (Finep), que vem desenvolvendo de programa de apoio à inovação, no âmbito do complexo da saúde desde 2004.

No campo acadêmico, cabe destacar os trabalhos desenvolvidos pelo grupo de pesquisa e inovação em saúde da Fiocruz, liderado por Carlos Gadelha (2003, 2007, 2011, 2012), sobre a dinâmica produtiva e de inovação do Ceis. Sobre a política nacional de ciência e tecnologia

\footnotetext{
${ }^{9}$ As ações do Gecis contemplam: i) a política específica de desoneração e de retorno a TEC de fármacos e medicamentos estratégicos; ii) a proposta de anteprojeto de lei de compras governamentais voltado para o fortalecimento da produção brasileira; iii) Cooperação entre o Ministério da Saúde e o Ministério do Desenvolvimento, indústria e Comércio Exterior para melhoria da qualidade da produção nacional e iv) a aprovação de proposta de consulta pública, elaborada pelo GT de Regulamentação de Biofármacos a ser submetida ao Colegiado da Anvisa.
}

Rev. Adm. Pública - Rio de Janeiro 49(4):915-936, jul./ago. 2015 
em saúde, vale destacar as contribuições de Reinaldo Guimarães (2004, 2005, 2006), assim como as de José Eduardo Cassiolato e Helena Lastres (1999, 2007), sobre as especificidades do Sistema Nacional de Inovação em Saúde no Brasil. Também de forma explícita, a sensibilização do campo da saúde coletiva ao potencial da base produtiva da saúde como indutor de desenvolvimento econômico e social tomou forma no congresso da Associação Brasileira de Saúde Coletiva (Abrasco) de 2012, cujo tema foi "Saúde é Desenvolvimento: ciência para cidadania".

Abordagens críticas à priorização do Ceis na agenda governamental também foram desenvolvidas dentro do campo acadêmico. Autores como Trevisan e Junqueira (2010a, 2010b) consideram, por exemplo, que a prioridade da política nacional de saúde deveria ser a definição de um novo padrão de consumo em saúde, incorporando as inovações tecnológicas em todas as entidades da federação, a partir do município até a União, e não o inverso. Segundo os autores:

Por mais relevante que seja, a transformação tecnológica não é o ponto central no estabelecer de um novo perfil de consumo de saúde porque não há condições orçamentárias para distribuir inovação tecnológica em toda a capilaridade do sistema, em especial se este sistema é pensado em termos de uma operação em rede. É na cooperação técnica, especialmente na difusão das formas de domínio das inovações, com ações articuladas entre a União e os estados que, na ponta do sistema, no município, ocorre o novo padrão de consumo em saúde propiciado pelos avanços tecnológicos. (Trevisan e Junqueira, 2010a:648)

Apesar da existência de análises dissonantes, pode-se verificar que o conjunto dos atores envolvidos no fluxo político da inserção do Ceis na agenda governamental atua na disseminação de uma determinada percepção do papel da saúde no desenvolvimento nacional.

Essa "imagem política" (Baumgartner e Jones, 1993) constitui um instrumento de comunicação acerca da implementação da política pública, conferindo legitimidade à mesma. De acordo com Capella (2012:113):

Para que um problema chame a atenção do governo é preciso que uma imagem, ou um consenso em torno de uma política, efetue a ligação entre o problema e uma possível solução. A criação de uma imagem é considerada um componente estratégico na mobilização da atenção do microssistema em torno de uma questão.

Assim, a solução do problema da fragilidade da base produtiva da saúde passa pela adoção de políticas de investimento nos setores estratégicos da mesma, especialmente os mais intensivos em tecnologias, denominados "portadoras de futuro" (Gadelha et al., 2012). Mas para que essa solução se concretize é necessária a atuação de atores políticos dentro e fora do governo, assim como de um contexto macropolítico favorável, conforme postulado no modelo de Kingdon (2003). Esses elementos pertencem ao fluxo político. 


\subsection{0 fluxo político}

Em termos institucionais, a introdução do Ceis nas prioridades da agenda de desenvolvimento nacional traz uma novidade a partir da aproximação do Ministério da Saúde das instâncias deliberativas do Plano Brasil Maior, sob a coordenação geral do MDIC. Esse movimento culminou no dia 20 de setembro de 2011, quando o ministro da Saúde foi convidado a fazer parte do Conselho Nacional de Desenvolvimento Industrial ${ }^{10}$ do Plano Brasil Maior, presidido pelo ministro do Desenvolvimento, Indústria e Comércio Exterior, Fernando Pimentel. A aproximação do Ministério da Saúde, portanto, da área social, com aqueles tradicionalmente voltados para a gestão econômica (a exemplo dos ministérios e autarquias mencionados nessa análise) é representativa da evolução nas políticas de saúde no período recente. ${ }^{11}$ Trata-se de um duplo movimento de valorização do potencial da base produtiva da saúde para alavancar o desenvolvimento nacional, e de inclusão das prerrogativas do Ministério da Saúde nos objetivos da principal política de desenvolvimento do país. Nesse sentido, a rede de governança do Plano Brasil Maior reflete a inovação de se pensar de forma sistêmica as variáveis sociais e econômicas na agenda de desenvolvimento nacional, a partir de uma abordagem integrada da saúde.

O reconhecimento da importância da base produtiva da saúde na agenda de desenvolvimento nacional também se beneficiou da atuação de "empreendedores políticos", fortemente envolvidos nos distintos encontros, reuniões e seminários visando à aproximação das instituições, das expertises sobre o complexo da saúde e da política industrial como um todo. A título de exemplo, podemos citar o envolvimento da Secretaria de Ciência, Tecnologia e Insumos Estratégicos do Ministério da Saúde, que vem defendendo a importância e fortalecimento da base produtiva da saúde desde o começo dos anos 2000, assim como o mencionado MDIC, no âmbito de sua Secretaria de Desenvolvimento da Produção, o Ministério da Ciência, Tecnologia e Inovação ou ainda o BNDES. Esses empreendedores tiveram a oportunidade de articular uma estratégia comum em favor da priorização do complexo da saúde dentro do projeto de desenvolvimento nacional, com o objetivo de fortalecer os produtores públicos e a infraestrutura de produção e inovação em saúde do setor público. Ademais, recorrendo à abordagem

\footnotetext{
${ }^{10}$ Segundo informações do MDIC: o Conselho Nacional de Desenvolvimento Industrial (CNDI), formado por 13 ministros de Estado, pelo presidente do BNDES e por 14 representantes da sociedade civil, é a instância superior de aconselhamento institucional do Plano Brasil Maior. Cabe ao conselho estabelecer as orientações estratégicas gerais, subsidiando as atividades do sistema de gestão. Disponível em: <www.brasilmaior.mdic.gov.br/conteudo/158>. Acesso em: fev. 2013.

${ }^{11}$ Cabe destacar que as antigas políticas de fomento ao complexo da saúde não se beneficiavam desta intersetorialidade: a exemplo da mencionada Política Industrial e Tecnológica e de Comércio Exterior e da política para o desenvolvimento produtivo foram construídas sob a égide de instituições voltadas para a administração econômica e a política de ciência e tecnologia (MDIC, MCT, MF, MPOG, MAPA, MIN, MME,MS, Apex-Brasil, BNDES, CNPq, Finep; de forma análoga, o Mais Saúde (informalmente conhecido como o PAC Saúde) foi desenvolvido de forma autônoma e, portanto, desarticulada com o setor econômico envolvido, pelo Ministério da Saúde.
} 
desenvolvida por Kingdon (2003), que ressalta a importância do papel do presidente no processo de definição das políticas públicas, cabe destacar a mensagem ao Congresso Nacional de 2013 (Brasil, 2013); divulgada pela presidente do país, a referida mensagem destaca especificamente a importância do complexo industrial da saúde para o desenvolvimento nacional. Dessa forma, além do comprometimento dos empreendedores de política pública, cabe destacar o engajamento da presidente da República para o sucesso do Plano Brasil Maior, como revela esta citação, extraída do discurso proferido durante a cerimônia de instalação dos Conselhos Setoriais de Competitividade do Plano Brasil Maior:

O Brasil está se preparando para um salto de competitividade em sua economia. Para isso é necessário um desenvolvimento acelerado de nossas capacidades científicas e tecnológicas. O Brasil também está se preparando para ter, diante do acirramento das crises e de processos recessivos na economia internacional, uma política pró-cíclica de investimento. Tenham certeza de que meu governo estará sempre ao lado da produção e do emprego no Brasil. (Brasil 2013)

Enfim, a atuação dos empreendedores de política pública, mesmo beneficiando-se do apoio presidencial, é tributária do ambiente macropolítico no qual ela se insere, ou, no vocabulário de Kingdon (2003), do "humor nacional". Os dois mandatos do presidente Lula e a atual administração da presidente Dilma Rousseff foram marcados por uma política voluntarista visando aliar investimentos públicos para sustentar o crescimento econômico e fortalecimento das políticas sociais. O comprometimento do governo com a proposta "desenvolvimentista" reflete-se no empreendedorismo dos funcionários da alta administração que foram capazes de materializar este projeto, característico da "institucionalidade neodesenvolvimentista", notadamente a partir do segundo mandato do presidente Lula (Machado et al., 2010:2374). Do ponto de vista macroeconômico, cabe mencionar que a primeira década dos anos 2000 foi marcada por uma das maiores crises da história econômica, cujo epicentro foram os países industrializados. O Brasil, nesse contexto, beneficiou-se do superávit de sua balança comercial liderado pelo crescimento vertiginoso do preço das commodities no começo dos anos 2000 e do dinamismo de seu mercado interno para conter esse choque externo (Carvalho e Kupfer, 2011). Esse conjunto de fatores revelou-se favorável à manutenção e ao aumento dos investimentos públicos para sustentar o crescimento econômico do país com foco na inclusão social. Incluir o complexo econômico-industrial da saúde nas prioridades do projeto de desenvolvimento nacional constituiu, dessa forma, uma decisão coerente com o "humor nacional" contemporâneo.

A análise destes três fluxos permite a aplicação da caracterização do complexo da saúde ao modelo de fluxos contínuos, conforme demonstra a figura 2, que sintetiza o processo de tomada de decisão que levou à inserção do complexo industrial da saúde nas prioridades do Plano Brasil Maior. 
Figura 2

\section{A introdução do complexo industrial da saúde nas prioridades do Plano Brasil Maior nos fluxos contínuos de Kingdon (2003)}

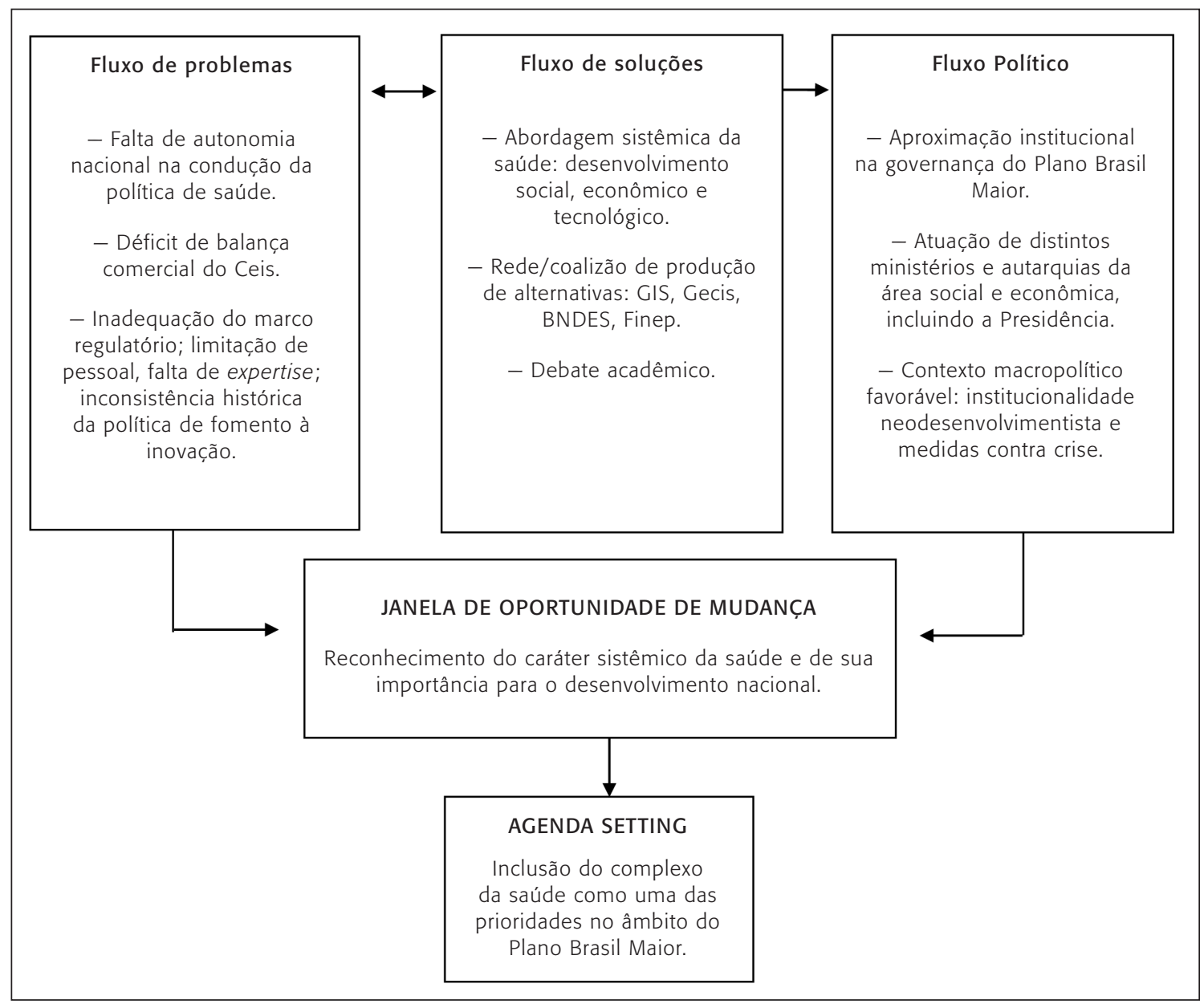

Fonte: Elaboração própria com base em Capela (2012:98).

\section{Considerações finais}

O SUS configura-se como um dos maiores sistemas de saúde universal do mundo. A despeito disso, a insuficiência de recursos disponível para o mesmo, assim como a composição da origem de seu financiamento (majoritariamente privada) e a dependência dos mercados internacionais para adquirir insumos estratégicos de saúde em decorrência da baixa capacidade de produção e de geração, uso e difusão de inovação impõem diversas barreiras para a efetivação 
de um sistema universal, integral e equânime, conforme previsto na Carta Magna (1988) e nas leis orgânicas da Saúde (Leis nos 8.080 e 8.142, ambas de 1990).

De acordo com o modelo de fluxos múltiplos, a decisão de inserir o Ceis nas prioridades do Plano Brasil Maior é fruto da junção de elementos oriundos dos fluxos de problemas, de soluções e políticos. O diagnóstico de fragilidade da base produtiva da saúde, o desenvolvimento de uma percepção sistêmica da mesma, a conjuntura macropolítica favorável e a atuação de empreendedores políticos com apoio presidencial contribuíram para essa tomada de decisão. Ao analisar esse processo, deve-se também levar em consideração que os determinantes da tomada de decisão política envolvem elementos diversos, várias vezes oriundos da avaliação de programas anteriores, evidenciando um fenômeno de interligação entre os distintos ciclos da política pública, conforme descrito por Jobert (1995).

No caso da saúde, essa afirmação é ilustrada pela inclusão da saúde em programas anteriores de governo, a exemplo dos mencionados nesta análise. Ressalta-se, dessa forma, a importância da constituição e da atuação de redes e coalizões de política pública, articulando atores de origens diversas: acadêmicos, membros do governo, representantes da esfera privada, entre outros. E, por fim, verifica-se o avanço no reconhecimento do caráter sistêmico da ação pública contemporânea no que se refere à base produtiva da saúde no Brasil, no sentido da integração entre os objetivos econômicos e sociais, de desenvolvimento tecnológico e de expansão da proteção social, de política industrial e de política de saúde. Nas palavras de Augusto (1989:2):

As distinções rígidas entre os aspectos "econômicos" e "sociais" da ação governamental não têm vigência no processo real. Nem aqueles nem estes são, verdadeira ou inteiramente, uma coisa ou outra. Antes, é na maneira pela qual tais dimensões estão articuladas que se expressa o fundamento da sociabilidade em ato.

Ressalte-se, entretanto, que a decisão de incluir o complexo econômico industrial da saúde nas prioridades do Plano Brasil Maior representa tão somente o começo do ciclo de execução da política industrial de saúde no âmbito desse programa. Nesse sentido, vale chamar atenção para a importância que deve ser atribuída ao processo de formulação das políticas para o desenvolvimento dessa base produtiva, à implementação das mesmas, e, posteriormente, às suas avaliações, de maneira a garantir que a integração dos objetivos sociais e econômicos se efetue de maneira efetiva.

Ademais, como uma sugestão de agenda de estudo, ressalta-se a necessidade de integrar a lógica territorial ao planejamento da política industrial em saúde como um todo, com vistas à formulação de políticas diferenciadas segundo necessidades regionais e à realização de investimentos para a redução das desigualdades em saúde; conforme ressaltam Machado e colaboradores (2010:2381), em um país com dimensões continentais, heterogêneo e desigual, como o Brasil, estas se apresentam como questões críticas para "uma prática transformadora da realidade social". 


\section{Referências}

ABREU, Matias W.; NEIVA, Vinícius M.; LIMA, N. Modelos de tomada de decisão no processo orçamentário brasileiro: uma agenda de pesquisa. Revista do Serviço Público, Brasília, v. 63, n. 2, p. 135-155, abr./jun. 2012.

ALBUQUERQUE, Eduardo M.; CASSIOLATO, José E. As especificidades do sistema de inovação do setor saúde: uma resenha da literatura como introdução a uma discussão sobre o caso brasileiro. Estudos FeSBE, Belo Horizonte, v. 1, 2000. Disponível em: < www.amxinformatica.com.br/redesist2/ images/projeto_saude/textos/documentos/material_bibliografico/Albuquerque_E._M._Cassiolato_J.E._As_especificidades_do_sistema.pdf>. Acesso em: 21 jan. 2013.

AUGUSTO, Maria H. O. Políticas públicas, políticas sociais e política de saúde: algumas questões para reflexão e debate. Rev. Sociol. USP, São Paulo, v. 1, n. 2, p. 105-119, 1989.

BAUMGARTNER, Frank R.; JONES, Bryan D. Agendas and instability in American politics. Chicago: University of Chicago Press, 1993.

BRASIL. Discurso da presidenta da República, Dilma Rousseff, durante cerimônia de anúncio de novas medidas do Plano Brasil Maior e instalação dos Conselhos Setoriais de Competitividade. Brasília/DF, 2012. Disponível em: <www2.planalto.gov.br>. Acesso em: 4 fev. 2013.

BRASIL. Plano Brasil Maior 2011/2014 Inovar para competir. Competir para crescer. 2011. Disponível em: <www.brasilmaior.mdic.gov.br/publicacao/recursos/arquivos/biblioteca/Apresentacao_Plano Brasil Maior_-_port_rev_abril_2012.pdf>. Acesso em: 15 fev. 2013.

BRASIL. Presidente. Mensagem ao Congresso Nacional. $3^{\text {a }}$ Sessão Legislativa Ordinária da 54a Legislatura. Brasília: Presidência da República, 2013.

CAPELLA, Ana Cláudia N. Perspectivas teóricas sobre o processo de formulação de políticas públicas. In: HOCHMAN, Gilberto; ARRETCHE, Marta; MARQUES, Eduardo (Org.). Políticas públicas no Brasil. Rio de Janeiro: Fiocruz, 2012.

CAPELLA, Ana Cláudia N. Perspectivas teóricas sobre o processo de formulação de políticas públicas. Revista Brasileira de Informações Bibliográficas em Ciências Sociais, v. 61, p. 25-53, 2006.

CAPELLA, Ana Cláudia N. The trajectory of public management policy in the governamental agenda: Lula and FHC administrations. Temas em Administração, Araraquara, v. 2, n. 2, p. 1-14, 2008. Disponível em: $<$ www.google.com $/$ url sa $=t \& r c t=j \& q=\&$ esrc $=s \&$ source $=$ web\&cd $=1 \& v e d=-$ 0CB0QFjAA\&url=http\%3A\%2F\%2Fseer.fclar.unesp.br\%2Ftemasadm\%2Farticle\%2Fdownload\%2F6122\%2F4596\&ei=OE7OVImgGfHgsASqx4DwBw\&usg=AFQjCNE01SdJ3Omc3dTgyBxh4mlVUB9X8g\&sig2=sjo3yFdk2QylD11Fxc1fBQ\&bvm=bv.85076809,d.cWc>. Acesso em: 7 mar. 2013.

CARVALHO, Laura; KUPFER, David. Diversificação ou especialização: uma análise do processo de mudança estrutural da indústria brasileira. Revista de Economia Política, v. 31, n. 4 (124), p. 618 637, out./dez. 2011. 
CASSIOLATO, José Eduardo; LASTRES, Helena M. Inovação, globalização e as novas políticas de desenvolvimento industrial e tecnológico. In: CASSIOLATO, José E. (Org.). Informação e globalização na era do conhecimento. Rio de janeiro: Campus, 1999. p. 164-190.

CASSIOLATO, José Eduardo; LASTRES, Helena M. Inovação e sistemas de inovação: relevância para a área de saúde. Reciis - Revista Eletrônica de Comunicação, Informação e Inovação em Saúde, Rio de Janeiro, v. 1, n. 1, p. 153-162, jan./jun. 2007.

CIMOLI, Marco et al. Instituições e políticas moldando o desenvolvimento industrial: uma nota introdutória. Revista Brasileira de Inovação, Rio de Janeiro, v. 6, n. 1, p. 55-85, jan./jun. 2007.

COHEN, Michael D.; MARCH, James G.; OLSEN, Johan P. A Garbage can model of organizational choice. Administrative Science Quarterly, v. 17, n. 1, p. 1-25, mar. 1972.

FLEXOR, George; LEITE, Sergio P. Análise de políticas públicas: breves considerações teórico-metodológicas. In: LEITE et al. Contradições gerenciais na disseminação da "cultura corporativa": o caso de uma estatal brasileira. Rev. Adm. Pública, Rio de Janeiro, v. 40, n. 3, p. 357-84, maio/jun. 2006.

FREY, Klaus. Políticas públicas: um debate conceitual e reflexões referentes à prática da análise de políticas públicas no Brasil. Planejamento e políticas públicas, n. 21, jun. 2000. Disponível em: <www.ipea.gov.br/pub/ppp/ppp21/Parte5.pdf>. Acesso em: 11 fev. 2013.

GADELHA, Carlos A. G. Complexo econômico-industrial da saúde: produtos e insumos estratégicos para as políticas e programas de saúde. Brasília: Conass, 2007.

GADELHA, Carlos A. G. O complexo industrial da saúde e a necessidade de um enfoque dinâmico na economia da saúde. Ciência e Saúde Coletiva, v. 8, n. 2, p. 521-535, 2003.

GADELHA, Carlos A. G.; COSTA, Lais S. Saúde e desenvolvimento no Brasil: avanços e desafios. Revista de Saúde Pública, v. 6, n. Supl., p. 13-20, 2012.

GADELHA, Carlos A. G.; COSTA, Lais S.; VIANA, Ana L. D. O complexo econômico-industrial da saúde e o desenvolvimento nacional. Revista Princípios, n. 114, p. 5-10, 2011.

GADELHA, Carlos A. G., MALDONADO, José; COSTA, Lais S. O complexo produtivo da saúde e sua relação com o desenvolvimento: um olhar sobre a dinâmica da inovação em saúde. In: GIOVANELLA, Lígia et al. (Org.). Políticas e sistemas de saúde no Brasil. Rio de Janeiro: Fiocruz, 2012. p. 209-238.

GADELHA, Carlos A. G. et al. A dinâmica do sistema produtivo da saúde: inovação e complexo econômico-industrial. Rio de Janeiro: Fiocruz, 2012.

GOMIDE, Alexandre A. Agenda governamental e formulação de políticas públicas: o projeto de lei de diretrizes da política nacional de mobilidade urbana. Texto para discussão no 1334, IPEA, Brasília, abr. 2008. Disponível em: <www.ipea.gov.br/portal/images/stories/PDFs/TDs/td_1334.pdf>. Acesso em: 23 jan. 2013.

GUILLÉN, Arturo R. A necessidade de uma estratégia alternativa de desenvolvimento no pensamento de Celso Furtado. In: SABOIA, João; CARVALHO, Fernando J. C. (Org.). Celso Furtado e o século XXI. Rio de Janeiro: Instituto de Economia da UFRJ, 2007. 
GUIMARÃES, Reinaldo. Bases para uma política nacional de ciência, tecnologia e inovação em saúde. Ciência \& Saúde Coletiva, v. 9, n. 2, p. 375-387, 2004.

GUIMARÃES, Reinaldo. Ciência, tecnologia e inovação: um paradoxo na reforma sanitária. In: LIMA, Nísia T. et al. Saúde e democracia: história e perspectivas do SUS. Rio de Janeiro: Fiocruz, 2005.

GUIMARÃES, Reinaldo. Pesquisa em saúde no Brasil: contexto e desafios. Rev. Saúde Pública, v. 40, n. Esp., p. 3-10, 2006.

IMS HEALTH. The global use of medicines: outlook through 2015. Disponível em: <http://www. imshealth.com/deployedfiles/ims/Global/Content/Insights/IMS\%20Institute\%20for\%20Healthcare\%20Informatics/Global_Use_of_Medicines_Report.pdf>. Acesso em: 3 abr. 2013.

JOBERT, Bruno. Rhétorique politique, controverses scientifiques et construction de normes institutionnelles: esquisse d'un parcours de recherché. In: FAURE, Alain; POLLET, Gilles; WARIN, Pierre. La construction du sens dans les politiques publiques. Paris: L'Harmattan, 1995. p. 13-24.

JOBERT, Bruno; MULLER, Pierre. L'État en action. Paris: Presses Universitaires de France, 1987.

JONES, Charles. An introduction to the study of public policy. Belmont: California Wadsworth Pub. Co., 1970.

KINGDON, John W. Agendas, alternatives, and public policies. 3. ed. Nova York: Harper Collins, 2003.

LASCOUMES, Pierre; LE GALÈS, Patrick. Sociologia da ação pública. Maceió: Edufal, 2012.

LASTRES, Helena M.; CASSIOLATO, José E. Inovação e sistemas de inovação: relevância para a área de saúde. Reciis - Revista Eletrônica de Comunicação, Informação e Inovação em Saúde, Rio de Janeiro, v. 1, n. 1, p. 153-162, 2007.

LOWI, Theodore J. Four systems of policy, politics and choice. Public Administration Review, v. 32, n. 4, p. 298-310, 1992.

MACHADO, Cristiani V. et al. O planejamento nacional da política de saúde no Brasil: estratégias e instrumentos nos anos 2000. Ciência \& Saúde Coletiva, v. 15, n. 5, p. 2367-2382, 2010.

MALDONADO José et al. A dinâmica inovativa do subsistema de base mecânica, eletrônica e de materiais. Rev. Saúde Pública, São Paulo, v. 46, n. Supl., p. 29-36, 2012.

MINISTÉRIO DA SAÚDE. Saúde dá preferência a produtos nacionais. Disponível em: <www.blog. saude.gov.br/saude-da-preferencia-a-produtos-nacionais/> . Acesso em: nov. 2012.

MINISTÉRIO DA SAÚDE. Saúde é estratégia para desenvolvimento industrial. Disponível em: <http:// portalsaude.saude.gov.br/portalsaude/index.cfm/?portal=pagina.visualizarNoticia\&codConteu$\mathrm{do}=2545 \&$ codModuloArea $=162 \&$ chamada $=$ saude-e-estrategica-para-desenvolvimento-industrial $>$. Acesso em: 22 nov. 2012.

NAGEM, Fernanda A.; SILVA, Sandro P. A inserção da economia solidária na agenda da política pública federal de geração de trabalho e renda. In: ENCONTRO INTERNACIONAL DE ECONOMIA SOLIDÁRIA FINANÇAS SOLIDÁRIAS E DESENVOLVIMENTO TERRITORIAL, VII, 2011, São Paulo.

Rev. Adm. Pública - Rio de Janeiro 49(4):915-936, jul./ago. 2015 
O ESTADO DE S. PAULO. A fila dos remédios. São Paulo, 26 nov. 2012.

THE WORLD MEDICAL MARKETS FACT BOOK. World leaders in health industry analysis. Londres: Espicom Business Intelligence, 2013.

TREVISAN, Leonardo; JUNQUEIRA, Luciano A. P. Gestão em rede do Sus e a nova política de produção de medicamentos. Saúde Soc., São Paulo, v. 19, n. 3, p. 638-652, 2010a.

TREVISAN, Leonardo; JUNQUEIRA, Luciano A. P. Rede social, pacto de gestão e o Programa Mais Saúde Direito de Todos 2008-2011. Economia Global e Gestão, v. 15, n. 3, p. 43-62, 2010b. Disponível em: <www.scielo.gpeari.mctes.pt/scielo.php?script=sci_pdf\&pi$\mathrm{d}=$ S0873-74442010000300004\&lng =pt\&nrm =iso\&tlng=pt $>$. Acesso em: 16 jan. 2013.

VARGAS, Marco A. et al. Inovação na indústria química e biotecnológica em saúde: em busca de uma agenda virtuosa. Rev. Saúde Pública, São Paulo, v. 46, n. Supl., p. 37-40, 2012.

VIDOTTI, Carlos C. F.; CASTRO, Lia L. C.; CALIL, Simone S. New drugs in Brazil: do they meet Brazilian public health needs? Rev. Panam. Salud Pública/Pan Am. J. Public Health, v. 24, n. 1, p. 36-45, 2008.

Antoine Metten é mestre em ciência política pelo Instituto de Estudos Políticos de Grenoble (IEPG), pesquisador do Grupo de Pesquisa de Inovação em Saúde (GIS) da Fundação Oswaldo Cruz (Fiocruz). E-mail: antoine.metten@gmail.com.

Laís Silveira Costa é doutora em saúde pública pela Escola Nacional de Saúde Pública Sergio Arouca da Fundação Oswaldo Cruz (Ensp/Fiocruz), mestre em development studies pela London School of Economics and Political Science (LSE) e pesquisadora do Grupo de Pesquisa de Inovação em Saúde (GIS) da Fiocruz. E-mail: achelais@hotmail.com.

Carlos Augusto Grabois Gadelha é economista, doutor em economia pelo Instituto de Economia da Universidade Federal do Rio de Janeiro (UFRJ); líder do Grupo de Pesquisa de Inovação em Saúde (GIS) e coordenador geral do mestrado profissional em Política e Gestão de Ciência, Tecnologia e Inovação em Saúde do departamento de Administração e Planejamento em Saúde da Escola Nacional de Saúde Pública Sergio Arouca da Fundação Oswaldo Cruz (Ensp/Fiocruz). Atualmente é secretário do Desenvolvimento da Produção do Ministério do Desenvolvimento, Indústria e Comércio Exterior (SDP/MDIC). E-mail: gadelha@ensp.fiocruz.br.

Jose Maldonado é doutor em engenharia da produção pela Universidade Federal do Rio de Janeiro (UFRJ); professor do departamento de Administração e Planejamento em Saúde da Escola Nacional de Saúde Pública Sergio Arouca da Fundação Oswaldo Cruz (Ensp/Fiocruz) e pesquisador do Grupo de Pesquisa de Inovação em Saúde (GIS) da Fiocruz. E-mail: jose.maldonado@ensp.fiocruz.br. 